\title{
MSCs modifies the proliferation of leukemia MOLT-4 cells and induces their apoptosis through up-regulating Bax, caspase-3, and-8, and down-regulating Bcl-2 expression
}

\author{
Mohaddeseh Rahbaran ${ }^{1)}$, Sadegh Shojaei Baghini ${ }^{2}$, Mahsa Mardasi ${ }^{3)}$, Ehsan Razeghian $^{4)}$ \\ 1) Animal Biotechnology Department, National Institute of Genetic Engineering and Biotechnology (NIGEB), Tehran, Iran \\ ${ }^{2)}$ Plant Biotechnology Department, National Institute of Genetic Engineering and Biotechnology (NIGEB), Tehran, Iran \\ ${ }^{3)}$ Plant Sciences and Biotechnology Department, Faculty of Life Sciences and Biotechnology, Shahid Beheshti University G. C., \\ Tehran, Iran \\ ${ }^{4)}$ Human Genetics Division, Medical Biotechnology Department, National Institute of Genetics Engineering and Biotechnology \\ (NIGEB), Tehran, Iran
}

\begin{abstract}
Currently, it has been suggested that human mesenchymal stem cells (MSCs), a well-known multipotent stem cells, could modify the biological process in leukemia cells. Therefore, we evaluated human MSCs' possible effects on apoptosis and proliferation of acute lymphoblastic leukemia (ALL) MOLT-4 cells. Accordingly, MOLT-4 cells were co-cultured with MSCs. Then, 24, 48, and 72 hours after treatment, the apoptosis percentages of co-cultured MOLT- 4 cell apoptosis was estimated using flow cytometry analysis. Also, cells proliferation rates were measured by MTT assay after 24, 48, and 72 hours of treatment. Finally, the expression ratio of candidate genes, including Bax, Bcl-2, and caspase-3, and -8, were evaluated in MOLT- 4 cells co-cultured with MSCs. Based on results, MSCs stimulated the apoptosis of MOLT- 4 cells after 48 and 72 hours but not 24 hours of treatment compared with the control group (MOLT-4 cells). Also, MSCs induced robust a reduction in MOLT-4 cell proliferation rate compared with the control group. On the other hand, Real-Time PCR results indicated a decrease in Bcl-2 expression, and conversely a promotion in Bax, and caspase-3, and -8 expression at mRNA levels. In sum, results signified that MSCs could exert anti-leukemic effects on leukemia MOLT-4 cells through promoting Bax/Bcl-2 ration concomitant with up-regulating caspases expression.
\end{abstract}

Keywords: Mesenchymal stem cells (MSCs), Acute lymphoblastic leukemia (ALL), MOLT-4 cells, Proliferation, Apoptosis

(Received April 12, 2021; Accepted April 22, 2021)

\section{INTRODUCTION}

Acute lymphoblastic leukemia (ALL) is an aggressive type of leukemia developed through the collecting of genomic lesions that disturb $\mathrm{T}$ or $\mathrm{B}$ cell growth $^{1-3)}$. ALL is the most shared form of childhood malignancies because 3 of 4 children who are recognized with leukemia suffering from $\mathrm{ALL}^{4}$. Indeed, ALL affects the lymphoid line of blood cells and is commonly described by the progress of large quantities of immature lymphocytes ${ }^{5,6}$.

Mesenchymal stem cells (MSCs) are well-known multipotent stromal cells that can differentiate into diverse types of human adult cell lineages ${ }^{7,8}$. They also have an important role in adjusting hematopoiesis and also sup-

Corresponding author: Ehsan Razeghian, Human Genetics Division, Medical Biotechnology Department, National Institute of Genetics Engineering and Biotechnology (NIGEB), Tehran, Iran. E-mail: ehsan.razeghi@gmail.com port the successful hematopoietic stem cell transplantation and enrich tumor stroma by several functions, such as producing the growth factors, improvement of the tumor vessel creation, and establishing niches for tumor stem cells $^{9-11)}$. Importantly, MSCs can affect immune cells by generating a myriad of soluble factors and also cell-to-cell contact ${ }^{12}$. Meanwhile, it seems that soluble factors produced by MSCs play a central role in immunomodulatory effects exerted by MSCs on immune mature cells. Recent findings have demonstrated that MSCs elicited suppressive effects on lymphoid lineage by producing various cytokines inducing cell cycle arrest at G0 and G1 phases ${ }^{13)}$. Besides, MSCs derived from patients suffering from leukemia commonly show diminished proliferative competence and also the ability for improving leukemic cell viability ${ }^{14)}$. Moreover, MSCs can affect leukemia cell biology, including apoptosis in normoxic and also hypoxic conditions ${ }^{15)}$. Other investigations have indicated that MSCs co-cultured with leukemia cells 
induced leukemic cell apoptosis and senescence by inducing caspase cascade activation and affecting GSK$3 \alpha / \beta$ and ERK1/2 signaling pathways ${ }^{16)}$. As well, MSCs could trigger a significant reduction in telomere length and also telomerase activity in leukemia K562 cells; on the other hand, MSCs affected K562 cells biological process through producing interleukin (IL)-6, IL-8, and transforming growth factor-beta (TGF- $\beta$ ) leading to the inhibition of $\beta$-catenin pathways concurrently improving tumor-suppressive functions of $\mathrm{p} 53^{17)}$.

Herein, we evaluated the apoptosis and proliferation rates of human leukemia MOLT- 4 cells when co-cultured with MSCs using Annexin V/PI staining followed by flow cytometry and MTT (3-[4,5-dimethylthiazol-2-yl]-2,5 diphenyl tetrazolium bromide) assay, respectively. Also, expression levels of the Bax, Bcl-2, and caspase-3 and -8 were measured using Real-Time PCR.

\section{MATERIALS AND METHODS}

\section{Cell culture}

The MOLT-4 cell line was obtained from Pasteur institute Cell Bank (Tehran, Iran) and then was cultivated in RPMI 1640 (Gibco, NY, USA) with 10\% fetal bovine serum (FBS) (Gibco, NY, USA). Also, bone marrow MSCs were obtained from Royesh Stem Cell Biotechnology Institute Cell Bank (Tehran, Iran) and were cultivated in Dulbecco's Modified Eagle Medium (DMEM) (Gibco, NY, USA) with $10 \%$ fetal bovine serum (FBS) (Gibco, NY, USA). Cells were kept at $37^{\circ} \mathrm{C}$ humidified atmosphere in $5 \% \mathrm{CO}^{2}$ incubator.

\section{Co-culture of MOLT-4 cells with BMMSCs}

Second passage MSCs were seeded in plates comprising DMEM at a density of $5 \times 10^{4}$ cells $/ \mathrm{cm}^{2}$. The medium was altered when the MSCs feeder layer reached confluence. Then, MOLT- 4 cells $\left(2 \times 10^{6}\right)$ were added into supernatant and then were incubated for 24,48 , and 72 hours.

\section{MTT assay}

MTT assay was applied to evaluate MSCs' effect on MOLT-4 cell proliferation. Accordingly, after 24, 48, and 72 hours of MSCs co-culturing with MOLT- 4 cells, $20 \mu \mathrm{l}$ of reagent (5 mg MTT/ml medium) was added into the wells containing MSCs plus MOLT- 4 cells and then cells were incubated at $37^{\circ} \mathrm{C}$ for $4 \mathrm{~h}$. After that, the optical density of wells was estimated at $570 \mathrm{~nm}$ with Epoch ELISA reader (BioTek, Winooski, Vermont). The viability of MOLT-4 cells co-cultured with MSCs was expressed as a percentage of the control samples viability (MOLT-4 cells). Values are means \pm SEM from triplicate tests.

\section{Apoptosis analysis using Annexin V-FITC/PI}

Apoptotic cells were recognized using AnnexinV and propidium iodide staining (BD Pharmingen, USA) considering the manufacturer's instructions. Firstly, on 24, 48, 72 hours upon treatment, the cells were washed twice with cold PBS followed by resuspension in $1 \mathrm{X}$ binding buffer at a concentration of $1 \times 10^{6}$ cells $/ \mathrm{ml}$. After that, $100 \mu$ lof the solution $\left(1 \times 10^{5}\right.$ cells $)$ was transferred to a $5 \mathrm{ml}$ culture tube. Then, $5 \mu \mathrm{l}$ of Annexin V-FITC and 5 $\mu l$ of PI were added followed by the addition of $400 \mu \mathrm{l}$ of $1 \mathrm{X}$ binding buffer. Then, cells were incubated for 15 $\min$ at RT $\left(25^{\circ} \mathrm{C}\right)$ in the dark. Finally, cells were evaluated by FACSCalibur flow cytometer (Becton-Dickenson, Mountain View, CA, USA) and FlowJo software.

\section{RNA extraction and cDNA synthesis}

Following discarding the medium from plates, MSCs were adherent and MOLT-4 cells were suspended. The leukemic cell's total RNA was isolated utilizing RNX Plus solution kit (Sinaclon, Tehran, Iran). Following cell lysis, the yields were kept at $-70^{\circ} \mathrm{C}$ and thawed when RNA isolation was required. High capacity kit (Bioneer, Alameda, CA) was employed for producing singlestranded complementary DNA (cDNA) from the isolated RNA.

\section{Gene expression analysis by real-time qPCR}

Real-time quantitative polymerase chain reaction (qRT-PCR) was carried out applying the SYBR Green reagent (Thermo Fisher Scientific). Relative gene expression was estimated by the Pfaffl method. The used primer pairs are listed in Table 1.

\section{Statistical analysis}

Data are illustrative of triplicate experiments and values are presented in mean \pm SEM. GAPDH was employed as the internal control. One-Way ANOVA test was accomplished to assess the observed statistical differences. All analyses were done utilizing the Graph Pad Prism software version 8.0.1. P values $<0.05$ were considered statistically significant.

Table 1 Primer pairs used for Real-Time PCR

\begin{tabular}{c|cl}
\hline Gene & \multicolumn{2}{c}{ Primer $\left(5^{\prime}-3^{\prime}\right)$} \\
\hline \multirow{2}{*}{ Caspase 3 } & F & TGAGCCATGGTGAAGAAGGA \\
& R & TCGGCCTCCACTGGTATTTT \\
\hline \multirow{2}{*}{ Caspase 8 } & F & CAGGCAGGGCTCAAATTTCT \\
& R & TCTGCTCACTTCTTCTGAAATCTGA \\
\hline \multirow{2}{*}{ Bcl-2 } & F & TCGCCCTGTGGATGACTGAG \\
& R & CAGAGTCTTCAGAGACAGCCAGGA \\
\hline \multirow{2}{*}{ Bax } & F & TTTGCTTCAGGGTTTCATCC \\
& R & GCCACTCGGAAAAAGACCTC \\
\hline \multirow{2}{*}{ GAPDH } & F & TGATGACATCAAGAAGGTGGTGAAG \\
& R & TCCTTGGAGGCCATGTGGGCCAT \\
\hline
\end{tabular}



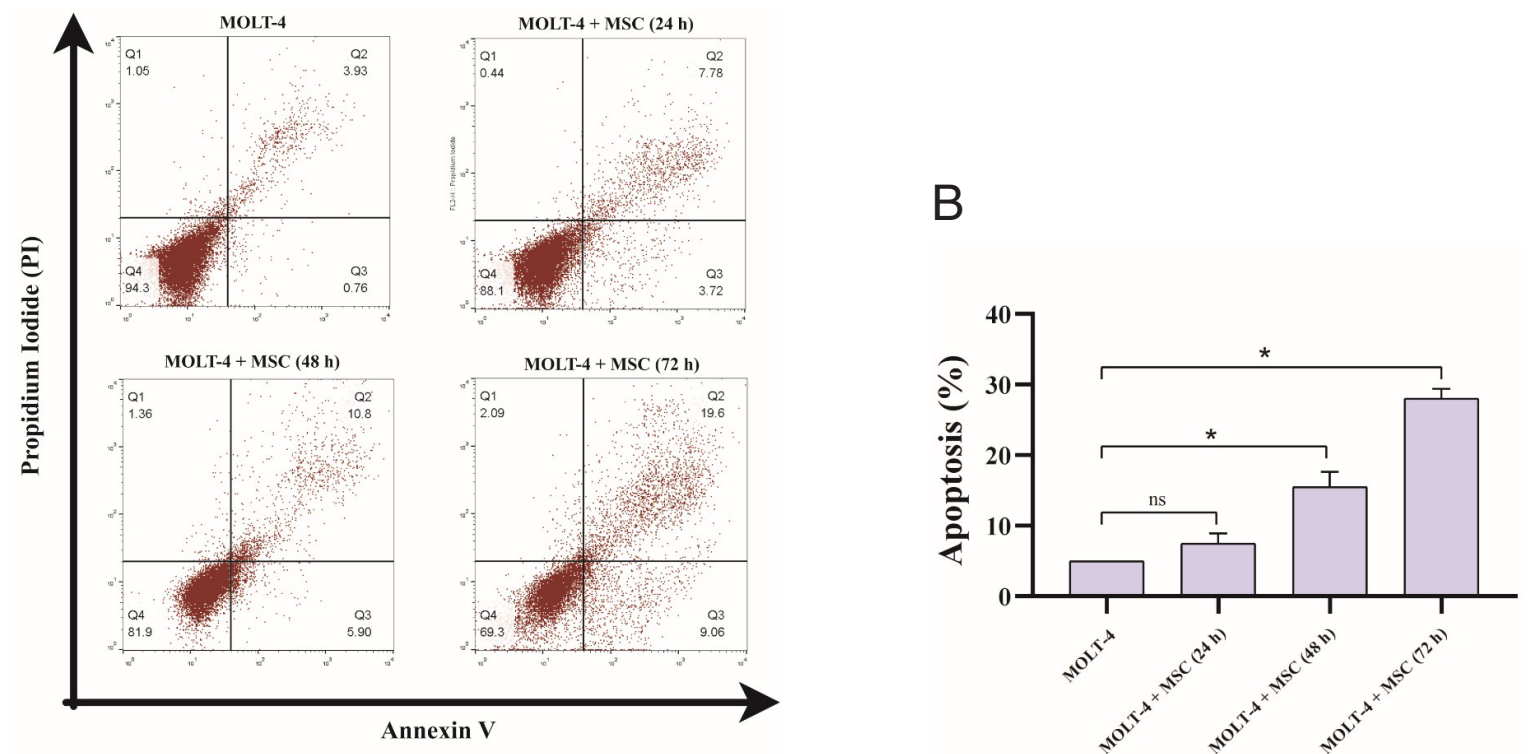

Fig. 1 The effects of MSCs on the apoptosis of MOLT-4 cells. Figure 1A demonstrates a representable sample of measurement of the apoptosis of MOLT-4 cells co-cultured with MSCs using Annexin-V/PI staining after 24, 48, and 72 hours of treatment. The results show the triplicate tests. The percentage of cells in each quadrant is demonstrated (viable cells are in Q4). Figure 1B presents the percentages of apoptotic cell of MOLT-4 cells co-cultured with MSCs after 24,48 , and 72 hours of treatment. Data are exposed as means \pm SEM of triplicate tests. One-Way ANOVA test was accomplished to assess the observed statistical differences. P-value $<0.05$ was considered statistically significant. $\left({ }^{*} ; p<0.05\right.$, ns; nonsignificant)

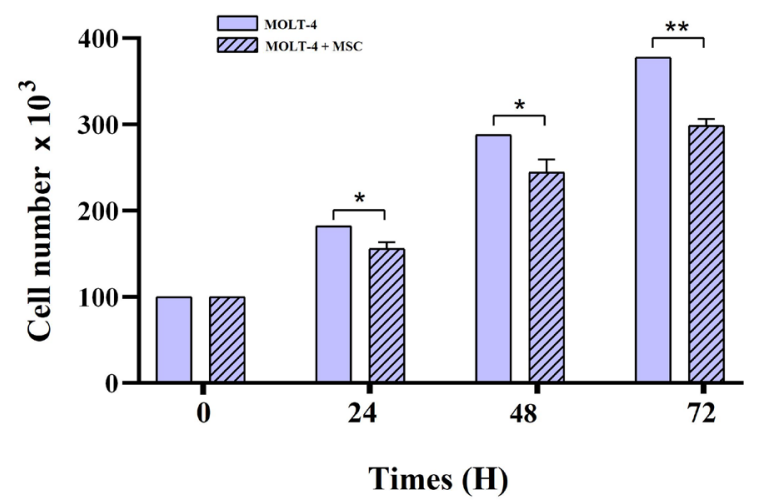

Fig. 2 The effects of MSCs on the proliferation of MOLT4 cell. The figure shows the proliferation rates of MOLT- 4 cells when these cells were co-cultured with MSCs for 24,48 , and 72 hours of treatment estimated by MTT assay. Data are exposed as means \pm SEM of triplicate tests. One-Way ANOVA test was accomplished to assess the observed statistical differences. P-value $<0.05$ was considered statistically significant. $\left({ }^{*} ; p<0.05,{ }^{* *} ; p<0.01\right)$

\section{RESULTS}

\section{MSCs co-cultured with MOLT-4 cells induced their apoptosis}

MOLT- 4 cells were co-cultured with MSCs to determine the possible effects of the MSCs on apoptosis of MOLT- 4 cell within 24, 48, and 72 hours of co- culturing. Based on FACS results, MSCs induced apoptosis in leukemic MOLT-4 cells during 24-72 hours of exposure compared with the control group (MOLT-4 cells). However, percentages of apoptotic cell were not significant in 24 hours of exposure, while it was significant in 48 and 72 hours of exposure compared with the control group (MOLT-4 cells) $(\mathrm{P}>0.05)$ (Figs. 1A, 1B). Accordingly, apoptosis percentages in MOLT- cells, MOLT- 4 cells co-cultured with MSCs during 24 hours of co-culturing, MOLT-4 cells co-cultured with MSCs during 48 hours of co-culturing, and finally MOLT- 4 cells co-cultured with MSCs during 72 hours of co-culturing were $4.08 \pm 1.19,8.72 \pm 2.35,16.74 \pm 3.11$, and $28.19 \pm 2.70 \%$ of total cells, respectively (Figs. 1A, 1B).

\section{MSCs co-cultured with MOLT- 4 cells abrogated their proliferation}

In the other step, leukemia MOLT-4 cells were cocultured with MSCs to evaluate the possible suppressive effects of the MSCs on MOLT- 4 cell proliferation within 24, 48, and 72 hours of co-culture. The result of MTT assay showed that MSCs reduced the proliferation rate of MOLT- 4 cell within 24, 48 , and 72 hours of co-culturing exposure compared with the control group (MOLT-4 cells) $(\mathrm{P}>0.05)$ (Fig. 2). Based on the analysis, the inhibitory effects of the MSCs on MOLT-4 cell proliferation were more obvious within 72 hours of co-culturing exposure than 24 and 48 hours of it $(\mathrm{P}>0.05)$ (Fig. 2). 
A

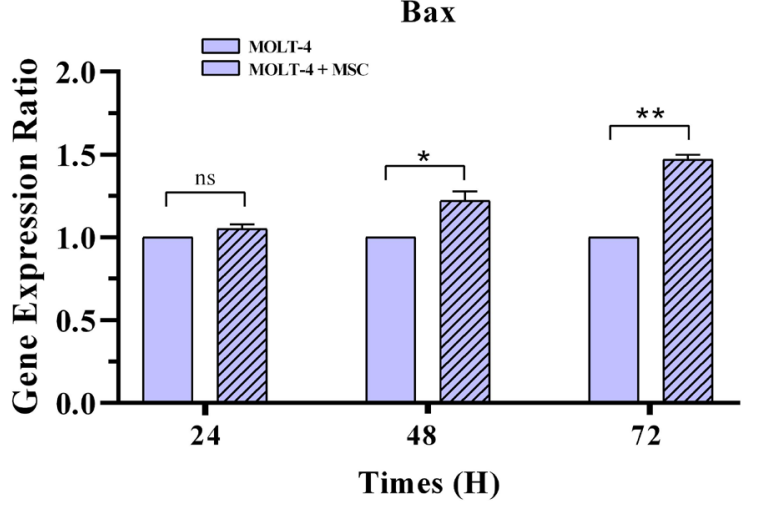

C

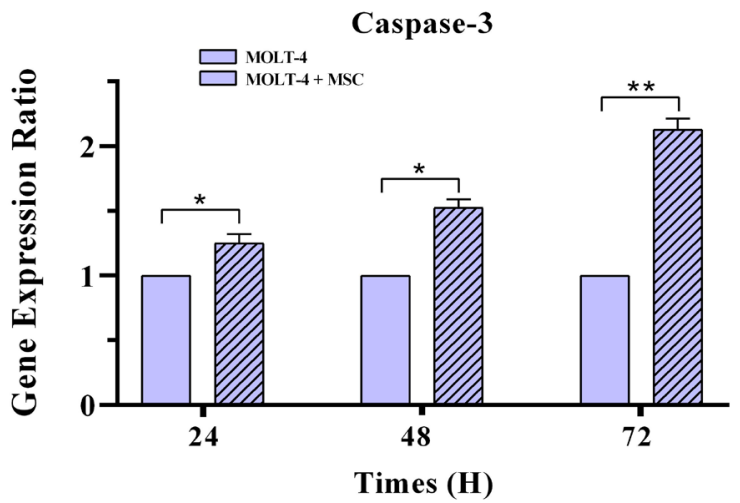

$\mathrm{B}$

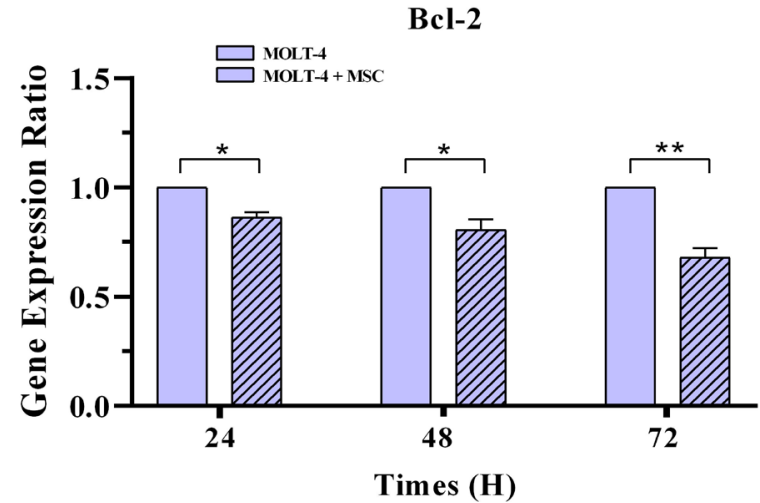

D

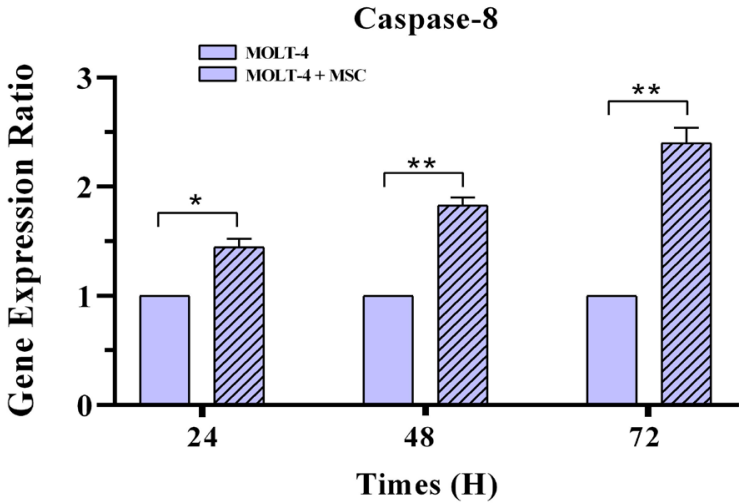

Fig. 3 Real Time-PCR data for Bax (A), and Bcl-2 (B), caspase-3 (C), and caspase-8 (D) expression in MOLT-4 cells cocultured with MSCs cells after 24, 48, and 72 hours of treatment. Data are exposed as means \pm SEM of triplicate tests. One-Way ANOVA test was accomplished to assess the observed statistical differences. GAPDH gene was used as the internal control. P-value $<0.05$ was considered statistically significant. ( ${ }^{*} ; p<0.05,{ }^{* *} ; p<0.01$, ns; non-significant)

\section{MSCs improved Bax/Bcl-2 ratio in MOLT-4 cells}

Investigation of the Bax gene expression in MOLT4 cells co-cultured with MSCs using Real-Time PCR displayed that Bax expression improved by 24,48 , and 72 hours of co-culturing exposure compared with the control group (MOLT-4 cells). Although the improvement was not significant within 24 hours of exposure, results verified significant and robust enhancement in Bax expression in MOLT-4 cells co-cultured with MSCs for 48 and 72 hours co-culturing $(\mathrm{P}>0.05)$ exposure compared with the control group (MOLT-4 cells) (Fig. 3A). Moreover, the improvement was more prominent within 72 hours of exposure than 24 and 48 hours of exposure (P $>0.05)$ (Fig. 3A).

On the other hand, the Bcl-2 expression ratio was attenuated in MOLT-4 cells compared with the control group during 24, 48, and 72 hours of exposure $(\mathrm{P}>0.05)$ (Fig. 3B). The reduction rate was higher in 72 hours of exposure than 24 and 48 hours of co-culture $(\mathrm{P}>0.05)$ (Fig. 3B).

\section{MSCs enhanced caspase-3 and caspase-8 expression in MOLT- 4 cells}

Measuring the possible effects of the MSCs on the expression rates of caspase- 3 and caspase- 8 in MOLT4 cells revealed that their expression ratio was strongly promoted in MOLT-4 cells during all periods of the exposure (P > 0.05) (Figs. 3C, 3D). As well, it was found that MSCs improved caspase- 3 and -8 expressions more prominent within 72 hours than 24 and 48 hours of exposure. Besides, analysis confirmed that MSCs enhanced caspase- 8 expression more obvious than caspase- 3 expression compared with the control group (Figs. 3C, 3D).

\section{DISCUSSION}

Drug resistance constructs a dilemma in treatment development in the context of leukemia therapy. Therefore, studies associated with the mechanisms corresponding to drug resistance, the molecular pathways contributed to leukemic cell apoptosis, and alternate treatments have attracted great attention ${ }^{18-20}$. Among diverse types of therapeutic modalities, MSCs are of superior interest because of their potential clinical utility ${ }^{21,22)}$. Therapies 
including MSCs are presenting growing promise in cancer therapy, while consequences have been unsatisfying because of the heterogeneity of MSC populations ${ }^{23-25)}$. During the last years, MSCs-elicited effects on various types of cancer, including hematologic malignancies, their mechanisms, and also sources along with their advantages and disadvantages have been $\operatorname{argued}^{26)}$. Several suggested mechanisms are citing the effects of MSCs in hematologic malignancies, but the most commonly believed mechanism is that MSCs could stimulate tumor cell cycle arrest ${ }^{27-29)}$. Consequently, identifying MSCs' dual roles in leukemic cell proliferation and apoptosis is of paramount importance. Recognizing the activities of MSCs in leukemia may be appropriate to hematologic cancer treatment. Though few studies have suggested that MSCs can straight improve the proliferation or apoptosis of malignant cells, the main theory is that MSCs inhibit both proliferation and apoptosis ${ }^{30,31)}$. Therefore, the efficacy of the MSCs application in the treatment of leukemia is poorly determined. Recent results have established that MSCs provoked inhibitory impacts on lymphoid lineage by generating several cytokines stimulating cell cycle arrest at G0 and G1 phases ${ }^{13)}$. Further, MSCs could modify leukemia cells' biological processes, such as apoptosis in normoxic and also hypoxic conditions ${ }^{15)}$. As well, MSCs co-cultured with leukemia cells could promote leukemic cell apoptosis and senescence by triggering caspase cascade activation and targeting survivalinvolved signaling pathways ${ }^{16,32)}$. Besides, MSCs could induce marked attenuation in telomere length and also telomerase activity in leukemia cells, and further modify these cells' biological process by generating IL-6, IL-8, and TGF- $\beta^{17)}$. In the present study, we evaluated MSC's effects on leukemia MOLT-4 cell proliferation as well as apoptosis once co-cultured together. Results showed that MSCs could induce MOLT-4 cell apoptosis after 48 and 72 hours of exposure. Also, we found that MSCs stimulated robust suppressive effects on MSCs proliferation. Real-time PCR analysis indicated that anti-leukemia effects of the MSCs were exerted by up-regulating proapoptotic protein Bax, and also caspase-3, and -8, concurrently down-regulating anti-apoptotic protein Bcl-2 expression at mRNA levels.

In sum, we suggested that MSCs could elicit marked anti-leukemia effects in the leukemic cell through suppressing anti-apoptotic proteins and also improving apoptosis intrinsic and extrinsic pathways; however, execution of large-scale studies in human subjects is of paramount importance.

Conflict of interest: There is no conflict of interest.

\section{References}

1) Hassanzadeh A., Naimi A., Hagh M. F., Saraei R., Marofi F. and Solali S. (2019) Kaempferol improves TRAIL-Mediated apoptosis in leukemia MOLT-4 cells by inhibition of anti-apoptotic proteins and promotion of death receptors expression. Anti-cancer agents in medicinal chemistry.

2) Saraei R., Marofi F., Naimi A., Talebi M., Ghaebi M., Javan N., Salimi O. and Hassanzadeh A. (2018) Leukemia therapy by flavonoids: Future and involved mechanisms. Journal of cellular physiology.

3) Hassanzadeh A., Farshdousti Hagh M., Alivand M. R., Akbari A. A. M., Shams Asenjan K., Saraei R. and Solali S. (2018) Downregulation of intracellular anti-apoptotic proteins, particularly cFLIP by therapeutic agents; the novel view to overcome resistance to TRAIL. Journal of cellular physiology. 233:6470-85.

4) Arora R. S. and Arora B. (2016) Acute leukemia in children: A review of the current Indian data. South Asian journal of cancer. 5:155-60.

5) Hassanzadeh A., Hosseinzadeh E., Rezapour S., Vahedi G., Haghnavaz N. and Marofi F. (2019) Quercetin promotes cell cycle arrest and apoptosis and attenuates the proliferation of human chronic myeloid leukemia cell line-K562 through interaction with HSPs (70 and 90), MAT2A and FOXM1. Anti-Cancer Agents in Medicinal Chemistry (Formerly Current Medicinal ChemistryAnti-Cancer Agents). 19:1523-34.

6) Mohaddeseh R. and Ehsan R. (2021) Anti-leukemic effects of the quercetin on human leukemia U937 cells mediated by down-regulation of Mcl-1, survivin, and XIAP. Annals of Cancer Research and Therapy. 29:55-61.

7) Markov A., Thangavelu L., Aravindhan S., Zekiy A. O., Jarahian M., Chartrand M. S., Pathak Y., Marofi F., Shamlou S. and Hassanzadeh A. (2021) Mesenchymal stem/stromal cells as a valuable source for the treatment of immune-mediated disorders. Stem cell research \& therapy. 12:1-30.

8) Iwamoto S., Mihara K., Downing J. R., Pui C. H. and Campana D. (2007) Mesenchymal cells regulate the response of acute lymphoblastic leukemia cells to asparaginase. The Journal of clinical investigation. 117:1049-57.

9) Marofi F., Hassanzadeh A., Solali S., Vahedi G., Mousavi Ardehaie R., Salarinasab S., Aliparasti M. R., Ghaebi M. and Farshdousti Hagh M. (2019) Epigenetic mechanisms are behind the regulation of the key genes associated with the osteoblastic differentiation of the mesenchymal stem cells: The role of zoledronic acid on tuning the epigenetic changes. Journal of cellular physiology.

10) Tavakoli S., Ghaderi Jafarbeigloo H. R., Shariati A., Jahangiryan A., Jadidi F., Jadidi Kouhbanani M. A., Hassanzadeh A., Zamani M., Javidi K. and Naimi A. (2020) Mesenchymal stromal cells; a new horizon in regenerative medicine. Journal of cellular physiology. 235:9185-210.

11) Lin W., Huang L., Li Y., Fang B., Li G., Chen L. and Xu L. (2019) Mesenchymal stem cells and cancer: clinical challenges and opportunities. BioMed Research International. 2019.

12) Shariati A., Nemati R., Sadeghipour Y., Yaghoubi Y., Baghbani R., Javidi K., Zamani M. and Hassanzadeh A. (2020) Mesenchymal stromal cells (MSCs) for neurodegenerative disease: A promising frontier. European journal of cell biology. 99:151097.

13) Baharaghdam S., Yousefi M., Movasaghpour A., Solali S., Talebi M., Ahani-Nahayati M., Lotfimehr H. and Shamsasanjan K. (2018) Effects of Hypoxia on Biology of Human Leukemia T-cell Line (MOLT-4 cells) Co-cultured with Bone Marrow Mesenchymal Stem Cells. Avicenna journal of medical biotechnology. 10:62-8.

14) Corradi G., Baldazzi C., Ocadlikova D., Marconi G., Parisi S., Testoni N., Finelli C., Cavo M., Curti A. and Ciciarello M. (2018) Mesenchymal stromal cells from myelodysplastic and acute myeloid leukemia patients display in vitro reduced proliferative potential and similar capacity to support leukemia cell survival. Stem cell research \& therapy. 9:271.

15) Ejtehadifar M., Shamsasenjan K., Movassaghpour A., Akbarzadehlaleh P., Dehdilani N., Abbasi P., Molaeipour Z. and Saleh M. (2015) The Effect of Hypoxia on Mesenchymal Stem Cell 
Biology. Advanced pharmaceutical bulletin. 5:141-9.

16) Fathi E. and Vietor I. (2021) Mesenchymal Stem Cells Promote Caspase Expression in Molt-4 Leukemia Cells Via GSK-3 $\alpha / \mathrm{B}$ and ERK1/2 Signaling Pathways as a Therapeutic Strategy. Current gene therapy. 21:81-8.

17) Fathi E., Valipour B., Sanaat Z., Nozad Charoudeh H. and Farahzadi R. (2020) Interleukin-6, -8, and TGF- $\beta$ Secreted from Mesenchymal Stem Cells Show Functional Role in Reduction of Telomerase Activity of Leukemia Cell Via Wnt5a/ $\beta$-Catenin and P53 Pathways. Advanced pharmaceutical bulletin. 10:307-14.

18) Wong R. S. (2011) Mesenchymal stem cells: angels or demons? Journal of biomedicine \& biotechnology. 2011:459510.

19) Trimarco V., Ave E., Facco M., Chiodin G., Frezzato F., Martini V., Gattazzo C., Lessi F., Giorgi C. A. and Visentin A. (2015) Crosstalk between chronic lymphocytic leukemia (CLL) tumor B cells and mesenchymal stromal cells (MSCs): implications for neoplastic cell survival. Oncotarget. 6:42130.

20) Hamid Reza Ghaderi J., Sedigheh S. and Abbas Zakeri B. (2021) Flavonoids kaempferol (KAE) and quercetine (QUE) inhibited proliferation of human leukemia THP-1 cells by up regulation of proapoptotic protein Bax and caspase 3/8 expression and down regulation of anti-apoptotic proteins Bcl-2, Bcl-xl and Mcl-1 expression. Annals of Cancer Research and Therapy. 29:41-6.

21) Ahmed H., Salem A., Atta H., Ghazy M. and Aglan H. (2014) Do adipose tissue-derived mesenchymal stem cells ameliorate Parkinson's disease in rat model? Human \& experimental toxicology. 33:1217-31.

22) Aqmasheh S., Shamsasanjan K., Akbarzadehlaleh P., Pashoutan Sarvar D. and Timari H. (2017) Effects of Mesenchymal Stem Cell Derivatives on Hematopoiesis and Hematopoietic Stem Cells. Advanced pharmaceutical bulletin. 7:165-77.

23) Capelli C., Pedrini O., Valgardsdottir R., Da Roit F., Golay J. and Introna M. (2015) Clinical grade expansion of MSCs. Immunology letters. 168:222-7.

24) Lyu T., Wang Y., Li D., Yang H., Qin B., Zhang W., Li Z., Cheng C., Zhang B. and Guo R. (2021) Exosomes from BM-MSCs promote acute myeloid leukemia cell proliferation, invasion and chemoresistance via upregulation of S100A4. Experimental Hematology \& Oncology. 10:1-13.
25) Zuo S., Sun L., Wang Y., Chen B., Wang J., Ge X., Lu Y., Yang N. and Shen P. (2021) Establishment of a novel mesenchymal stem cell-based regimen for chronic myeloid leukemia differentiation therapy. Cell death \& disease. 12:1-15.

26) Fathi E., Sanaat Z. and Farahzadi R. (2019) Mesenchymal stem cells in acute myeloid leukemia: a focus on mechanisms involved and therapeutic concepts. Blood Res. 54:165-74.

27) Zhu N., Wang H., Wei J., Wang B., Shan W., Lai X., Zhao Y., Yu J. and Huang H. (2016) NR2F2 regulates bone marrow-derived mesenchymal stem cell-promoted proliferation of Reh cells. Molecular medicine reports. 14:1351-6.

28) Lin H. D., Fong C. Y., Biswas A., Choolani M. and Bongso A. (2016) Human Umbilical Cord Wharton's Jelly Stem Cell Conditioned Medium Induces Tumoricidal Effects on Lymphoma Cells Through Hydrogen Peroxide Mediation. Journal of cellular biochemistry. 117:2045-55.

29) Abreu S. C., Xisto D. G., de Oliveira T. B., Blanco N. G., de Castro L. L., Kitoko J. Z., Olsen P. C., Lopes-Pacheco M., Morales M. M., Weiss D. J. and Rocco P. R. M. (2019) Serum from Asthmatic Mice Potentiates the Therapeutic Effects of Mesenchymal Stromal Cells in Experimental Allergic Asthma. Stem cells translational medicine. 8:301-12.

30) Brenner A. K., Nepstad I. and Bruserud O. (2017) Mesenchymal Stem Cells Support Survival and Proliferation of Primary Human Acute Myeloid Leukemia Cells through Heterogeneous Molecular Mechanisms. Frontiers in immunology. 8:106.

31) Kim N., Nam Y. S., Im K. I., Lim J. Y., Lee E. S., Jeon Y. W. and Cho S. G. (2015) IL-21-Expressing Mesenchymal Stem Cells Prevent Lethal B-Cell Lymphoma Through Efficient Delivery of IL-21, Which Redirects the Immune System to Target the Tumor. Stem cells and development. 24:2808-21.

32) Bozok Cetintas V., Aktug H., Oltulu F., Keskinoglu A., Erer Del Castello B. and Taskiran D. (2014) The effects of mesenchymal stem cells on lymphoblastic leukemia cell proliferation. Journal of BUON : official journal of the Balkan Union of Oncology. 19:1006-17. 